\title{
Research on the Dynamic Element Management Model of Experiment Project
}

\author{
Yang Xin/Doctroal \\ College of Tourism, Economics and Management \\ Chengdu University \\ Chengdu, China \\ 1960803040@qq.com
}

\author{
Zhang Chuanwu/Professor \\ College of Electrical and Information Engineering \\ Southwest Minzu University \\ Chengdu, China \\ zcw@swun.cn
}

\begin{abstract}
In order to overcome the weakness of manual management in the dynamic experiment project management, this paper presented the dynamic element management model of experiment project. The dynamic element management model of experiment project consists two parts. Analysis shows that the core elements are the equipments, experiment rooms and tutorials of the system while the relationships of these elements are the combination, correlation, or cooperation of these elements in system. This paper analyzed the elements of the experiment project from the viewpoint of the system theory, and assembled the key elements of the experiment project. The system set up the elements resource status library based on the usage status of the elements resource. The experiment project applicant request the elements resource needed by the experiment project while the system allocate the appropriate elements resource based on the status of the element resource library and the system policy, thus the system can service to the applicant of the experiment project. So that the dynamic element management model of experiment project can provide more fine-grain service on the elements resource combination of the system and it can increase the coefficient of utilization of the elements resource. That means this model can promote efficiency of the system.
\end{abstract}

Keywords-Experiment Project; Dynamic element Management; System Model; Element Resource Combination

\section{INTRODUCTION}

Teaching element consist the unit or element of the teaching activity, and it's the theory foundation of the discipline of pedagogy [1-3]. The element of experiment project is also the foundation of experiment project management. In order to overcome the weakness of manual management in the dynamic management of experiment project [4-7], encourage the student autonomous learning [810], and dynamically use the experiment elements to do innovation, we need study the dynamic element management model of experiment project.

There are many elements resource in the experiment project, but the elements resource of equipments, experiment rooms and tutorials are the most important elements in the implementation process of the students' experiment project. The dynamic element management system of experiment project utilizes information technologies to manage the key elements resource of the equipments, experiment rooms and tutorials in the fine-grain scale [11-13]. Because that the statistical use of the elements resource of the experiment project, the dynamic element management system of experiment project can configure the elements resource of equipments, experiment rooms and tutorials in more finegrained of the time slot to increase the coefficient of utilization of the elements resource, and promote the efficiency of the dynamic element management system of experiment project. This paper analyzes the elements resource of the system based on the system theory to find out the key elements resource of the system. Before the process of the system, the system set up the status library of the key elements resource of equipments, experiment rooms and tutorials based upon the usage of the elements resource. The applicant of the experiment project inquires the pre-allocation of the elements resource combination of the equipments, experiment room and tutorial in the appropriate time slot. Then the system combine the elements resource needed for the experiment project dynamically based on the status library, and allocate the combined elements resource of elements resource of equipments, experiment rooms and tutorials needed for the experiment project to the applicant. Thus the management system can ensure the applicant obtain the elements resource needed for the experiment project.

\section{THE ANALYSIS OF THE SYSTEM ELEMENT}

For a system, the boundary divides the system and its environments. So we must discern the system to its environment by define the boundary between the system and its environments. For the experiment project, it consists of the students, the text book or experiment tutorial books, tutorials, equipments and experiment devices, experiment rooms. The traditional teaching activity mainly based on the teachers instruction while the students just listen and practice just as the instruction. As the development of the discipline of pedagogy, there are more and more paying attention to the students' initiative. So the modern teaching encourage the student autonomous learning. The dynamic element management of experiment project can provide the high coefficient of utilization of the element resource and suit for the students' autonomous learning.

The interface of the dynamic element management model of experiment project is confined by the definition of the subject-object system. The subject of the dynamic element 
management model of experiment project is the students or applicants of the system, while the objects of the dynamic experiment system are the elements resource of the experiment project, in which the most important or key element are the equipments, experiment rooms and tutorials. When we use the information technology to assist to management experiment project, we aimed at the object of the system. Which means that the subject of the system can be the interface to the dynamic element management system of experiment project, so the system boundary may just include the object of the system. So that the factory of the dynamic element management system of experiment project mainly include the elements resource such as equipments, experiment rooms and tutorials.

The system process is that the applicant applies the elements resource needed for the experiment project while the system will allocate the elements resource to the applicant based upon the status of the element resource library and the system policy. In this process, the elements resource is the most critical element because of that the elements resource is limited contrast to the application of the applicant. The system allocates the elements resource is based upon the application of the applicant, the status of the element resource library, and the system policy. This is a dynamic process and depends on the behavior of the subjects and the usage of the objects. Due to the dynamic process of the elements resource, the statistical multiplexing usage of the elements resource can increase the coefficient of utilization of the elements resource dramatically, and thus we can achieve the aim of the dynamic experiment project management system: the maximum improvement of the system efficiency.

From the viewpoint of system constitution, the key elements resource of the dynamic element management model of experiment project includes equipments, experiment rooms and tutorials. The aim of the dynamic element management model of experiment project is the allocation of the limited elements resource. So the elements analysis is the foundation of the system model.

Firstly, for a system, we can denote the dynamic element management model of experiment project as a set specified as equation (1) from the integrity of the system from the system theory [14].

$$
S=\{E, R\}
$$

The elements of the system can be explicated list as the key elements resource of the system. So the dynamic element management model of experiment project can be denotes as the set of equipments, experiment rooms and tutorials list as set specified as equation (2).

$$
E=\left\{\begin{array}{lll}
E_{e} & E_{r} & E_{p}
\end{array}\right\}
$$

In the equation (2), the $E_{e} \quad E_{r} \quad E_{p}$ are corresponded to the equipments, experiment rooms and tutorials. We can elaborate the information of the elements resource of the dynamic element management model of experiment project as show in list 1.

TABLE I ELEMENTARY RESOURCE OF THE DYNAMIC ELEMENT

\begin{tabular}{|c|c|c|c|c|}
\hline \multirow[t]{2}{*}{ Elements } & \multicolumn{4}{|c|}{ Attributes } \\
\hline & Item & Num. & Time & Status \\
\hline \multirow{4}{*}{ Equipment } & $e_{1}$ & $\mathrm{~N}_{1}$ & $\mathrm{t}$ & $0 / 1$ \\
\hline & $e_{2}$ & $\mathrm{~N}_{2}$ & $\mathrm{t}$ & $0 / 1$ \\
\hline & $\cdots$ & $\ldots$ & $\ldots$ & $\ldots$ \\
\hline & $\mathrm{e}_{\mathrm{I}}$ & $\mathrm{N}_{\mathrm{I}}$ & $\mathrm{t}$ & $0 / 1$ \\
\hline \multirow{4}{*}{ Room } & $\mathrm{r}_{1}$ & $\mathrm{M}_{1}$ & $\mathrm{t}$ & $0 / 1$ \\
\hline & $r_{2}$ & $\mathrm{M}_{2}$ & $\mathrm{t}$ & $0 / 1$ \\
\hline & $\ldots$ & $\ldots$ & $\ldots$ & $\ldots$ \\
\hline & $\mathrm{r}_{\mathrm{J}}$ & $\mathrm{M}_{\mathrm{J}}$ & $\mathrm{t}$ & $0 / 1$ \\
\hline \multirow{4}{*}{ Person } & $\mathrm{p}_{1}$ & $\mathrm{~L}_{1}$ & $t$ & $0 / 1$ \\
\hline & $\mathrm{p}_{2}$ & $\mathrm{~L}_{2}$ & $\mathrm{t}$ & $0 / 1$ \\
\hline & $\cdots$ & $\cdots$ & $\cdots$ & $\cdots$ \\
\hline & $\mathrm{p}_{\mathrm{K}}$ & $\mathrm{L}_{\mathrm{k}}$ & $\mathrm{t}$ & $0 / 1$ \\
\hline
\end{tabular}
MANAGEMENT MODEL OF EXPERIMENT PROJECT

In list 1, there have I kinds of elements resource of equipment, while the ith equipment has $N_{\mathrm{i}}$ individuals. There have $\mathrm{J}$ experiment rooms and the jth experiment room can holds $M_{\mathrm{j}}$ students to deploy experiment project. And there have $\mathrm{K}$ tutorials and the kth tutorial can guide $L_{\mathrm{k}}$ students to implement the experiment project. Thus we can obtain the elements resource of the dynamic element management model of experiment project as set specified as equation (3):

$$
E=\left\{\begin{array}{lllllllll}
e_{1} & \cdot & e_{I} & r_{1} & \cdot & r_{J} & p_{1} & \cdot & p_{K}
\end{array}\right\}
$$

For the elements resource of the dynamic element management model of experiment project, they have corresponding status in the time dimension. That means for use of the elements resource, we can dive the time as time slot such as class 1 to $\mathrm{N}$ for a day, every element resource such as equipment, experiment room or tutorial, there are corresponding status for each time slot. The status of the elements resource denotes whether the elements resource are used in specified time slot, status 0 means that the element resource can be allocated to applicant while status 1 means that the element resource has allocated to other applicant. For the beginning of the system, all of the statuses are 0 , which means that all elements resource can be applied by students or applicants. When someone applied the elements resource on a time slot, then the status for the elements resource of the specified time slot is set to 1 , which means that the element resource of corresponding time slot has allocated to some applicant. 


\section{The CoOPERATION MECHANISM OF THE SYSTEM ELEMENTS RESOURCE}

In the equation (1), the $\mathrm{R}$ denotes the combination, correlation or cooperation mechanism. In the dynamic element management model of experiment project, the $\mathrm{R}$ denotes the combination allocation by applicant, which means that the system must combination or band the elements resource such as the equipments, experiment rooms and tutorials to the applicant.

For the elements resource of equipment, it can express as equation (4) in the form of set.

$$
E_{e}=\left\{e_{i} \mid i \in N_{i}\right\}
$$

In equation (3), there are $\mathrm{N}$ kinds of equipments, and the $\mathrm{i}$ kind of equipment has $N_{i}$ equipments. So that the number of the ith kind of equipment that the applicant inquired must in the field of $\left[\begin{array}{ll}1 & N_{i}\end{array}\right]$.

For the elements resource of experiment room, it can express as equation (5) in the form of set.

$$
E_{r}=\left\{r_{j} \mid j \in M_{j}\right\}
$$

In equation (5), there are $\mathrm{M}$ experiment rooms, and the jth kind of experiment room can holds $M_{j}$ applicants to implement its experiment. So that the number of the jth kind of experiment room that the applicant inquired must in the field of $\left[\begin{array}{ll}0 & M_{j}\end{array}\right]$ while the applicant may not need the experiment room.

For the elements resource of tutorial, it can express as equation (6) in the form of set.

$$
E_{p}=\left\{p_{k} \mid k \in L_{k}\right\}
$$

In equation (6), there are $L$ tutorial teachers, and the kth tutorial teacher can supervise $L_{k}$ applicants to implement its experiment. So that the number of the kth kind of tutorial teacher that the applicant inquired must in the field of $\left[\begin{array}{ll}0 & L_{k}\end{array}\right]$ while the applicant may not need the tutorial teacher.

When an applicant need implement an experiment project, the applicant must inquire to the dynamic element management system for the elements resource needed for the experiment project. The combination of the elements resource and the cooperation relationship can be express as equation (7).

$$
R=\left\{n_{i}\left(e_{i}\right) \quad m_{j}\left(r_{j}\right) \quad l_{k}\left(p_{k}\right) t\right\}
$$

In the equation (7), the number of the ith kind of equipment that the applicant inquired must in the field of $\left[\begin{array}{ll}1 & N_{i}\end{array}\right]$; the jth kind of experiment room that the applicant inquired must in the field of $\left[\begin{array}{ll}0 & M_{j}\end{array}\right]$; and the number of the kth kind of tutorial teacher that the applicant inquired must in the field of $\left[\begin{array}{ll}0 & L_{k}\end{array}\right]$. While the time $\mathrm{t}$ shows that the time slot that the applicant need the elements resource to carry the experiment project.

When the experiment project applicant request the elements resource needed by the experiment project. The system will allocate the appropriate elements resource based on the status of the element resource library and the system policy, then the system manager will set the status of the allocated elements resource as " 1 " which means it has been allocated and cannot used by others. If the applicant inquire successfully, then the applicant can use the elements resource in the appropriate time slot and must return the elements resource at the end of time slot.

The process of the dynamic element management system of experiment project may be described as following:

(1) When a student plan to implement a experiment project, he or she become the applicant.

(2) The applicant send the application message to the dynamic element management system of experiment project as () to apply the elements resource with specified time slot need by the experiment project.

(3) The dynamic element management system of experiment project inspect the elements resource library to find out the appropriate elements resource with the status as " 0 " to match the applied message.

(4) The dynamic element management system of experiment project allocate the corresponding elements resource to the applicant, and record the applicant ID number to the elements resource by bond them together.

(5) The dynamic element management system of experiment project set the status of the allocated element resource to " 1 ".

(6) The experiment operator prepare the corresponding elements resource to different applicants.

(7) The applicant get the elements resource from the experiment operator by its application confirm notice.

(8) The tutorial supervise the applicant student to carry out the experiment by using the equipments in experiment room.

(9) The applicant return the elements resource to the experiment operator.

(10) The experiment operator inspect the elements resource especially the equipments. If the elements resource is ok, then approve the applicant finish the experiment project. If there are any fault, then the experiment operator press the applicant to correct it. And go (10).

(11) End.

The step (1) to (11) form a loop control of the dynamic element management system of experiment project, and it can ensure the correct working of the dynamic element management system of experiment project. 


\section{CONCLUSION}

The dynamic element management model of experiment project is based on the set of elements resource. The system takes the set of elements resource of equipments, experiment rooms and tutorials as its core. And it takes the combination of the elements resource of equipments, experiment rooms and tutorials needed for the experiment project as its relationship. Thus the system can dynamically provide the service to the applicant by allocate the elements resource to specific applicant by under the condition of the status of the elements resource library and the combination need of elements resource of the applicant. Thus the three elements consists the model of dynamic element management system.

The system set up the elements resource status library based on the usage status of the elements resource. The experiment project applicant request the elements resource needed by the experiment project while the system allocate the appropriate elements resource based on the status of the element resource library and the system policy, thus the system can service to the applicant of the experiment project.

\section{ACKNOWLEDGMENT}

We thanks the support of the Teaching Innovation Project of Southwest Minzu University "Study on the Support System for the Quality of Teaching of Electrical and Information Discipline (2014)".

\section{REFERENCES}

[1] Dong Zhi-feng, Essential Teaching Factors and the Construction of Their Operating Patterns, Theory and Practice of Education, Vol.25, No.7, 2005, pp.52-55

[2] Li Dingren, Fan Zhaoxiong, The Optimization of the Teaching Factors and Teaching System, Education Science, Vol.19, No.6, Dec. 2003, pp.17-20

[3] Zhan Huifang, Mo Xiaoquan, The Changes of the Teaching Essence Factors and Their Relationships Under the Information Technology, Survey of Education, Vol.4, No.10, April 2015, pp.12-14

[4] Sun Tian-hao, Yang Meng-ning, Wang Qian, Zhu Zheng-yu, Huang Ren, Exploration on Open Innovative Experimental Management System Based on Experimental Items, Research and Exploration in Laboratory, Vol.34, No.8, 2015, pp.227-230

[5] Zhang Limin, The Project based Laboratory Applied in the Computer Teaching, University Education, April, 2014, pp.91-93

[6] Zhou Ying, primary exploration on the Project management of the Innovation Laboratory, Modern Education Equipment of China, 2009, No.5, pp.148-150

[7] Jin Wei-zu, Chen Xian-tao, Comprehensive Innovative Experiment Items in the Sustainable Computer Network, Research and Exploration in Laboratory, Vol.33, No.4, April 2014, pp.187-190, 236

[8] Sun Xiaoyong, Design and Implementation of Open Experimental Project Teaching Management System, Computer Era, No.12, 2015

[9] Li Xu-yun, Lu Yuan, Wang Meng-ling, Mei Ru-huan, Wang Hui-ping, Ying Song-min, Xia Qiang, On the Impact of Driven Experimental Teaching Mode on Studengs' Practice Motication, Research and Exploration in Laboratory, Vol.35, No.2, April 2016, pp.170-174

[10] Wang Gesi, Research and Practice of Open Experimental Teaching Platform of Analog Electronic Technology Course, Experimental Technology and Management, Vol.31, No.8, Aug. 2014, pp.170, 173

[11] Wang Cheng-Liang, Jiang Li, Fine-Grained Privilege Management Model and Its Application in B/S Application System, Computer System and Application, Vol.19, No.7, 2010, pp.79-82

[12] Zhao Wei-dong, Bi Xiao-qing, Lu Xin-ming, Design and Implemention of Fine-grained RBAC Model, Computer Engineering and Design, Vol.34, No.2, 2013, pp.474-479

[13] Wang Chao Liu Wei Yuan Pei-yuan, Research on the Parallel Processing Algorithm of STAP Based on Fine-grained Task Scheduling, Journal of Electronics \& Information Technology, Vol.34No., Jun. 2012, pp.1398-1403

[14] Sun Dongchuan, Lin Fuyong, Sun Kai, Introduction to System Engineering, Tsinghua University Press, 2009. Pp.11-13. 